\title{
A guaranteed obstacle avoidance guidance system
}

\section{The safe maneuvering zone}

\author{
Lionel Lapierre • Rene Zapata
}

Received: 13 January 2011 / Accepted: 2 December 2011 / Published online: 4 January 2012

(C) Springer Science+Business Media, LLC 2012

\begin{abstract}
This paper presents a practical solution to the guidance of a unicycle type robot, including path following, obstacle avoidance and the respect of wheeled actuation saturation constraint, without planning procedure. These results are based on an extension of previous results on path following control including actuation saturation constraints. New solution for obstacle avoidance, with guaranteed performance, is proposed.
\end{abstract}

Keywords Path following - Obstacle avoidance . Saturation constraints

\section{Introduction}

\subsection{Context and objectives}

The architecture of an autonomous mobile system can be seen as a collection of software processes, orchestrized within a software architecture and run on an hardware architecture. These processes can be roughly divided in 4 different groups, each of them realizing some basic robotic functions, cf. Fig. 1: Navigation, Guidance and Control, Mission Control and Communication management.

These basic robotic functions are then sequentially or contextually recruited to realize different type of behaviors, in order for the mobile system to efficiently operare in a complex and versatile environment. Some of these behaviors, providing decision making and planning capabilities

L. Lapierre $(\bowtie) \cdot R$. Zapata

LIRMM, 161, rue Ada, 34392 Montpellier, France

e-mail: lapierre@lirmm.fr

R. Zapata

e-mail: zapata@lirmm.fr

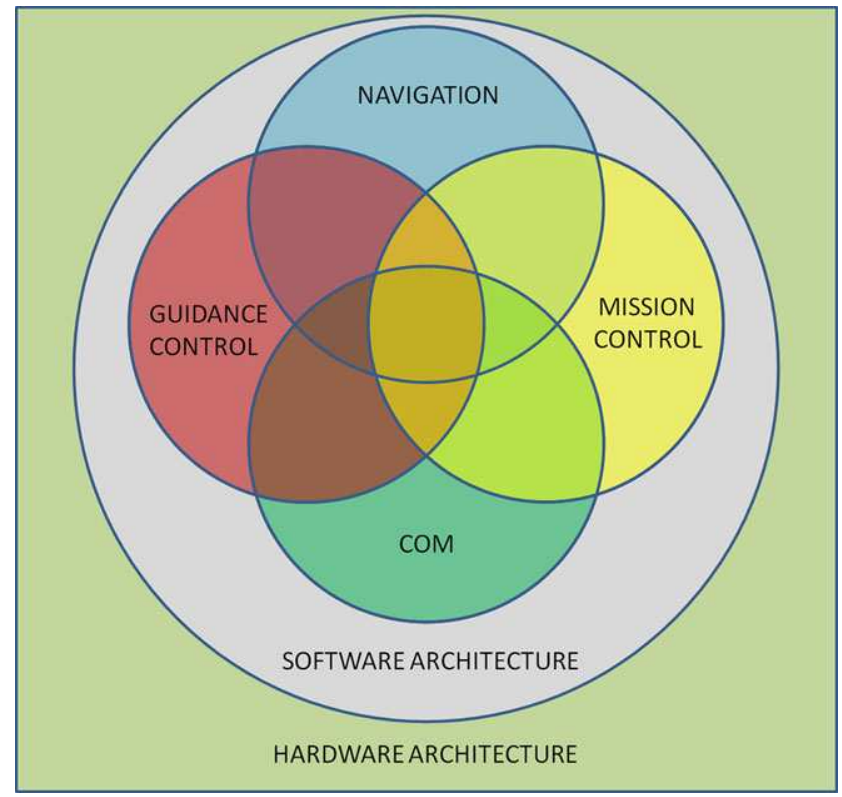

Fig. 1 Architecture of an autonomous mobile system

(Path planning, Mission Control, ...), are implemented at a high level of the architecture. Others, requiring rapid reaction, gain at being implemented at a lower level (Navigation, Guidance and Control, Reactive Obstacle Avoidance ...) and are generally run as periodical processes.

This paper focuses on the Reactive Guidance and Control subsystem which has to provide a periodical computation of the system reaction, in order to afford the system with permanent basic capabilities as path following, station keeping, obstacle avoidance, formation keeping...

Recent advances in nonlinear control theory (Lyapunov based design, backstepping...) provides the necessary tools to design Guidance and Control algorithms with guarantees of convergence. The Guidance system processes navigation 
data and path parameters in order to provide an achievable reference to the control system that drives the actuation. In this context, a guidance system should drive the system towards its current objective, while respecting the actuation saturation constraint, and providing a reference compatible with the system dynamics. This last point is not considered in this paper, and could be tackled using backstepping techniques (Krstić et al. 1995), assuming that the reference is differentiable, and is Globally, Uniformly and Asymptotically Convergent (GUAC). Moreover, this property warrants that, in the absence of sensor noise or modeling error, the control system will constantly and perfectly drive the system toward its objective. The effect of sensor noise will be to degrade the convergence property, down to a practical convergence, as far as the sensor suite and the navigation system afford an evaluation of the system states with a guaranteed and computable upper bound on the estimation error.

The objective of this paper is to propose a low-level reactive Guidance and Control subsystem that aims at preserving the convergence properties as the following functions are considered in the algorithm:

- Path following

- Actuation saturation constraint

- Obstacle avoidance

In Lapierre et al. (2006), we proposed a solution for path following control design of a unicycle type robot, that formally exhibits Global Uniform Asymptotic convergence (GUAC) property. In Lapierre and Indiverri (2007) this solution is extended to consider velocity saturation constraints of the actuators, while preserving the GUAC property. The solution proposed here, includes obstacle avoidance capability, combined with path following behavior. The objective is to propose a global solution to this problem, which formally guarantees:

- a Global Uniform Asymptotic Convergence of the robot to the path, when there is no obstacle, and

- that a minimum distance to the obstacle is respected, explicitly considering actuator velocity constraints, and

- that the system will retrieve its path following behavior when the obstruction has been avoided.

In the sequel, the Navigation system is considered as ideal and provides a perfect estimation of the system states, and the system models are considered as perfectly known.

\subsection{Some bibliographic elements}

\subsubsection{Path-following}

Motion control of autonomous vehicles has received considerable attention during the last few years. The solutions proposed in the literature can be roughly classified in three groups:
- point stabilization: the goal is to stabilize the vehicle at a given point, with a given orientation;

- trajectory tracking: the vehicle is required to track a time parameterized reference;

- path following: the vehicle is required to converge to and follow a path, without explicit temporal specifications.

Point stabilization presents a true challenge to control system designers when the vehicle has nonholonomic (or nonintegrable) constraints, since there is no smooth (or even continuous) state-feedback law that will yield stability, as pointed out by Brockett (1983). To overcome this difficulty three main approaches have been proposed: smooth time-varying control laws (Canudas de Wit et al. 1993; Godhavn and Egeland 1997 and Micaelli and Samson 1992) and discontinuous as well as hybrid feedback laws (Aguiar et al. 2000; Astolfi 1999; Hespanha 1996; Canudas de Wit and Sordalen 1992).

The trajectory tracking problem for fully actuated systems is now well understood and satisfactory solutions can be found in advanced nonlinear control textbooks. However, in the case of underactuated vehicles, that is, when the vehicle has less actuators than state variables to be tracked, the problem is still a very interesting topic of research. Linearization and feedback linearization methods (Walsh et al. 1994; Freund and Mayr 1997), as well as Lyapunov-based control laws (Canudas de Wit et al. 1993; Fierro and Lewis 1994) have been proposed.

Path following control has received relatively less attention than the other two problems. See the publications of Samson and Ait-Abderrahim (1991) and Micaelli and Samson (1993) for pioneering work in the area as well as Canudas de Wit et al. (1993) and Jiang and Nijmeijer (1999) and the references therein. Path following systems for marine vehicles have been reported by Encarnacao et al. (1992). The underlying assumption in path following control is that the vehicle's forward speed tracks a desired speed profile, while the controller acts on the vehicle orientation to drive it to the path. Typically, smoother convergence to a path is achieved, in comparison with the performance obtained with trajectory tracking controllers, and the control signals are less likely pushed to saturation. The work presented in this paper is based on the virtual target principle (Lapierre et al. 2006), where an added degree on freedom captures the motion of a virtual target on the path, allowing to relax the initial condition constraint exposed in Samson and AitAbderrahim (1991). Moreover the introduction of the virtual target will bring some advantages which will be exposed in the sequel.

\subsubsection{Inclusion of saturation constraint}

The abovementioned solutions of the literature provide controllers which may not be realistic, since they do not explicitly consider the effective actuators capacity, while they 
could require large actuator inputs to achieve convergence. An interesting study, applied to Swedish wheeled robot, can be found in Indiveri et al. (2009). The authors show that a saturated situation in the actuator induces an undesired coupling between the longitudinal and rotational velocities controls, loosing the possibility to exploit the advantageous holonomic property of the system. Hence, they propose a modification of the control expression, allowing to prioritize the execution of the basic robotic tasks (position and heading), in order to respect the saturation constraint on the actuators, while the tracking error converges to 0. Following a behavioral approach, this work has been extended to non-holonomic system in Arrichiello et al. (2009). The work presented here is based on Lapierre and Indiverri (2007), where the path following algorithm exposed in Lapierre et al. (2006) is extended to cope with actuation limitation, using saturating functions proposed in Jiang et al. (1998), with the objective of preserving the convergence property of the path following algorithm, while respecting at any time the saturation constraint of the actuators. Basic ideas are exposed in the sequel.

\subsubsection{Obstacle avoidance}

Obstacle avoidance strategy is another major issue to perform reliable applications. The system reaction can be computed as replanning function (Path replanning) or directly in the controller as a reflex behavior.

Planning a new path, free of potention collision, is still a major issue of the field. The reaction quantification is generally made according to an arbitrary positive potential field functions attached on obstacles that repels the robot, and an attractive field located on the goal. The main difficulty of this method is to design an artificial potential function without undesired local minima. Elnagar and Hussein (2002), propose to model the potential field by Maxwell's equations that completely eliminate the local minima problem, with the condition that an a priori knowledge of the environment is available. These methods are generally computationally intensive. Iniguez and Rossel (2002), proposes a hierarchical and dynamic method, that works on a non regular grid decomposition, simple and computationally efficient, both in time and memory. Ge and Cui (2000) address the problem of the adaptation of the potential field magnitude, in order to avoid the problematic situation where the goal is too close from an obstacle inducing an inefficient attractive field, called the Goals Non-Reachable with Obstacles Nearby. The work of Louste (1999) tackles the problem of coupling a path planning method, based on viscous fluid propagation, with nonholonomic robot kinematics restrictions. All these elegant solutions have clear advantages and the implementation of one of them is mandatory within the architecture, since they can provide a globally feasible path (if exists) that drives the system toward the objective. Nevertheless, planning a new path in a complex and versatile environment induces on the architecture a computational load that may not be compatible with a periodical recruitment, within a control period.

Another approach, based on a reflex behavior, is using the Deformable Virtual Zone (DVZ) concept, in which a robot kinematic dependent risk zone is located on the robot, surrounding it. The deformation of this zone is due to the intrusion of proximity information (obstacles within the risk zone). The system reaction is made in order to reform the risk zone to its nominal shape, implicitly going away from obstacles (Zapata et al. 2004). A combination of this method with the path following algorithm of Lapierre et al. (2006) is proposed in Lapierre et al. (2007). The limitation of this method is due of the virtual and arbitrary dynamics of the risk zone, that brings complexity in the control algorithm, and makes the whole system difficult to tune.

Another approach, classified as path deformation proposes to locally modify the shape of the path in order to contour the detected obstacle. Sgorbissa et al. (2010) propose a path deformation based on a Gaussian bell shape that allows for contouring the obstacle. In this present paper, we use a similar principle based on the definition of a Safe Maneuvering Zone (SMZ) originally introduced in Lapierre et al. (2010). As it will be exposed later, this solution uses the basic path following controller previously exposed, exploiting the convergence property of the algorithm.

It has to be noticed that the two last methods are local and cannot provide a global solution. On the other hand the planning methods are able to provide a global solution, if exists. This could be advantageously used within a planning process, that could be computationally intensive, and cannot be integrated at the reactive level. The solution is a combination of both types of methods. One is providing a path to be followed, free of obstacles as far as the environment knowledge allows for it, and the other allows for locally adapting the system trajectories in order to reactively avoid uncharted/misplaced/moving obstacles.

\section{Notation and basic models}

This section introduces the notation which is used in this paper. As a generic model for a nonholonomic mobile system, we consider a unicycle-type vehicle, of width $2 L$, equipped with 2 rear wheels of radius $R$ and a passive front wheel (see Fig. 2). The velocity of the center of mass of the robot is orthogonal to the rear wheels axis. Define $\{O\}$ an inertial (universal) frame and $\{B\}$ the body frame, attached to the vehicle onto the middle point of the rear wheels axis. Let $\mathbf{P}_{R}=[x, y]^{T}$ design the absolute coordinate of the origin of $\{B\}, \psi$ is the yaw angle of the vehicle. Let $u$ and $r$ denote the forward and rotational velocity of the vehicle, and 


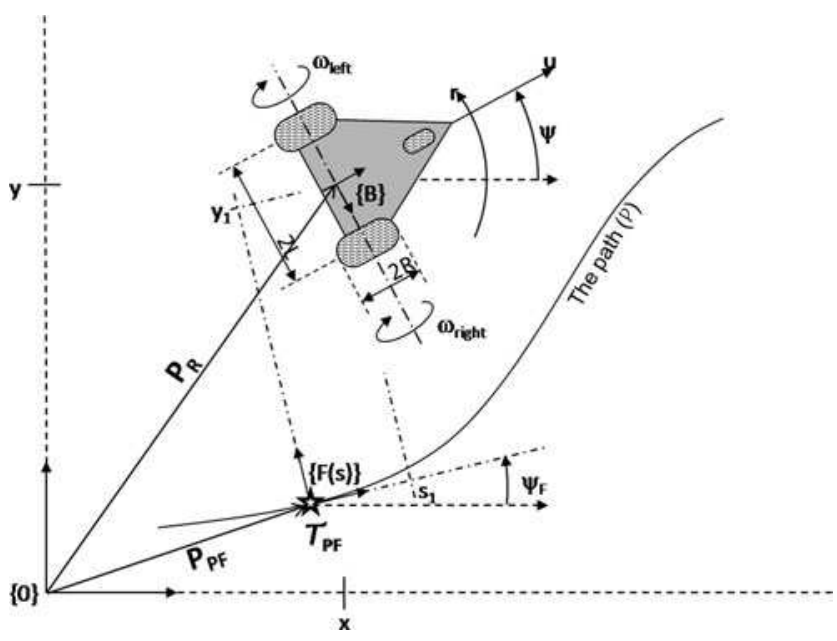

Fig. 2 Unicycle's parameters and frame definition

$w_{\text {left }}$ and $w_{\text {right }}$ express the wheels angular velocity, and the control inputs. The maximum achievable angular velocity of the wheels is denoted $w_{\max }$. Following this notation, the kinematic model of the unicycle is expressed as:

$\dot{x}=u \cos \psi$,

$\dot{y}=u \sin \psi$,

$\dot{\psi}=r$.

The expression of the actuation model is:

$w_{\text {left }}=(u-L r) / R$,

$w_{\text {right }}=(u+L r) / R$.

Define a path to be followed, denoted $\mathcal{P}$, and a SerretFrenet frame $\{F(s)\}$, attached on a point of the path defined by its curvilinear abscissa $s$. The orientation of $\{F\}$ with respect to $\{O\}$ is denoted as $\psi_{F}$. Assuming that the absolute coordinates of the origin of $\{F(s)\}$ is denoted by $\mathbf{P}_{P F}=\left[x_{F}(s), y_{F}(s)\right]^{T}, \mathcal{T}_{P F}(s)=\left[\mathbf{P}_{P F}, \psi_{F}\right]$ is defining the posture of $\{F(s)\}$, also called in the sequel path-followingtarget.

Clearly, the robot position $\mathbf{P}_{R}$ can be expressed as $[x, y]^{T}$ in $\{O\}$ or $\left[s_{1}, y_{1}\right]^{T}$ in $\{F(s)\}$. Let define the path curvature of the point located at $s$, as $c(s)$. The introduction of the variable $\theta=\psi-\psi_{F}$, and the combination with (1), yields to the kinematic model of the unicycle in $\{F\}$ as:

$\dot{s}_{1}=-\dot{s}\left(1-c y_{1}\right)+u \cos \theta$,

$\dot{y}_{1}=-c \dot{s} s_{1}+u \sin \theta$,

$\omega=r-c \dot{s}$

where $\omega=\dot{\theta}$.

Equation (3) captures the kinematic behavior of the unicycle system, expressed in the Frenet frame, attached to the path on a point described by its curvilinear coordinate $s .\left(s_{1}, y_{1}\right)$ are the coordinates of the robot in the Frenet frame $\{F\}$. Note that in this expression, we are using an added virtual degree of freedom, that is the path following

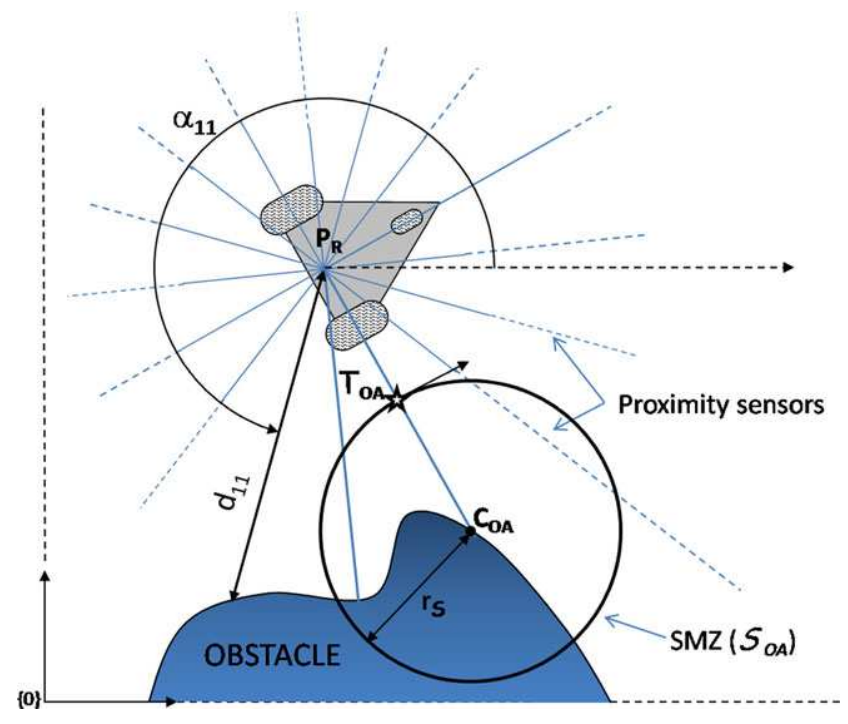

Fig. 3 Proximity sensors and safety maneuvering zone (SMZ) definition

virtual target evolution $\dot{s}$. As we will see in the sequel, and mathematically stated and solved in Lapierre et al. (2006), this procedure permits to solve the path following problem without restriction. Indeed, classically, for path following control design, the point to be reached on the path by the system is chosen as the closest point. This method has the major drawback of constraining the initial position of the robot in function of the maximum curvature of the path. This constraint is relaxed with the virtual target method reported in Lapierre et al. (2006).

In the following we use the subscript (.) $O A$ to design variables related to Obstacle Avoidance. For obstacle avoidance purpose, the robot is equipped with $m$ proximity sensors, pointing in $m$ different directions regularly distributed on the robot, and concurrently crossing onto the middle point of the rear wheels axis. These sensors periodically provide the distance to obstacle information as $\mathbf{d}=\left[d_{1}, d_{2}, \ldots, d_{m}\right]^{T}$ in the absolute direction $\alpha=\left[\alpha_{1}, \alpha_{2}, \ldots, \alpha_{m}\right]^{T}$. Clearly, $\alpha_{i}=$ $\psi+(2 \pi)(i / m)$, for $i=0, \ldots, m-1$. Consider the sensor that provides the smallest $d_{i}$, and define $\mathbf{C}_{O A}$ as the absolute position of that closest sensor/obstacle intersection. Define a safety maneuvering zone (SMZ), denoted $\mathcal{S}_{O A}\left(r_{\mathcal{S}}, \mathbf{C}_{O A}\right)$, as a circle of radius $r_{\mathcal{S}}$ centered in $\mathbf{C}_{O A}$, as shown in Fig. 3. Consider the point $\mathbf{P}_{O A}=\left[x_{O A}, y_{O A}\right]^{T}$ as the intersection between $\overline{\mathbf{P}_{R} \mathbf{C}_{O A}}$ and $\mathcal{S}_{O A}$. Define $\mathcal{T}_{O A}=\left[\mathbf{P}_{O A}, \psi_{O A}\right]$, called in the sequel obstacle-avoidance-target, where $\psi_{O A}$ will be defined later.

\section{Control design}

This section presents the methodology to design a low-level reactive Guidance and Control subsystem, that aims at pre- 
serving convergence properties as the following functions are considered in the algorithm:

- Path following

- Actuation saturation constraint

- Obstacle avoidance

\subsection{Path-following control design}

The solution to the problem of path following admits an intuitive explanation: a path following controller should look at (i) the distance from the vehicle to the path and (ii) the angle between the vehicle velocity vector and the tangent to the path, and reduce both to zero. This motivates the development of the kinematic model of the vehicle in terms of a Serret-Frenet frame $\{F\}$ that moves along the path; $\{F\}$ plays the role of the body axis of a path following target that should be tracked by the vehicle. Using this set-up, the abovementioned distance and angle become the coordinates of the error space $\left(s_{1}, y_{1}, \theta\right)$ where the control problem is formulated and solved, as expressed in (3).

Motivated by the work in Micaelli and Samson (1992), the Serret-Frenet frame $\{F\}$ that moves along the path to be followed is used with a significant difference: $\{F\}$ is not attached to the point on the path that is closest to the vehicle. Instead, the origin of $\{F\}$ along the path is made to evolve according to a conveniently defined function of time, effectively yielding an extra controller design parameter. As it will be seen, this seemingly simple procedure allows to lift the stringent initial condition constraints that arise with the path following controller described in Micaelli and Samson (1992), effectively warranting a global solution, i.e. whatever the initial conditions.

Equipped with the previously defined formalism, the problem under study is to design a kinematic control law, in terms of $\left[w_{\text {left }}, w_{\text {right }}, \dot{s}\right]$ that asymptotically drives a single unicycle robot to the path, with a desired arbitrary forward velocity profile $u_{d}$. We introduce the approach angle $\delta:=-\theta_{A} \tanh \left(k_{\delta} y_{1}\right)$, where $k_{\delta}$ is an arbitrary positive gain, and $\theta_{A}$ defines the asymptotic desired approach, which defines the approach angle when $\left|y_{1}\right|$ is huge. Traditionally, $\theta_{A}=\pi / 2$, which implies that the system is driven to the path with a relative incidence of $\pi / 2$, i.e. perpendicular to the path. Then, as shown in Lapierre et al. (2006), the following control law solves the path following problem

$$
\begin{aligned}
& w_{\text {left }}=\left(u_{d}-L r_{P F}\right) / R, \\
& w_{\text {right }}=\left(u_{d}+L r_{P F}\right) / R, \\
& \dot{s}=u \cos \theta+K_{s} s_{1}
\end{aligned}
$$

where

$r_{P F}:=\dot{\delta}-K_{1}(\theta-\delta)+c \dot{s}$

and $K_{1}$ and $K_{s}$ are arbitrary positive gains.
This solution is Globally Exponentially Convergent (GEC) and provides a useful decoupling between the heading and the forward velocity controllers. The reader is invited to refer to Lapierre et al. (2006) for complete proof, extension to dynamics and robustness.

Note that the guidance objective for path following is achieved when $\theta-\delta=0$, or equivalently when $\psi=\psi_{F}+\delta$. We introduce the global path following heading reference:

$\psi_{P F}:=\psi_{F}+\delta$.

\subsection{Inclusion of actuation saturation constraint}

The consideration of actuation constraint, within the previous path following strategy, has been treated in Lapierre and Indiverri (2007). It consists in adapting the current forward velocity reference according to the following constraint:

$\left\{\begin{array}{l}\left(w_{\text {max }}^{2}-w_{\text {left }}^{2}\right)>0, \\ \left(w_{\text {max }}^{2}-w_{\text {right }}^{2}\right)>0\end{array} \quad \forall t\right.$.

Proposition 1 The path following requirement, under actuation saturation constraint, is achieved using the following kinematic control:

$r_{\overline{P F}}=f_{r}+g_{r} u_{P F}$

where

$f_{r}=-k_{3} \tanh (\theta-\delta)+k_{4}(.) s_{1} c\left[1-s_{1} \delta^{\prime}\right]$,

$u_{\overline{P F}}=\frac{u_{d}}{u_{\max }} \frac{R w_{\max }-L\left(1 / 4+f_{r}^{2}\right)}{1+L\left(1 / 4+g_{r}^{2}\right)}$,

$g_{r}=c \cos \theta\left(1-s_{1} \delta^{\prime}\right)+\delta^{\prime} \sin \theta$,

$\delta^{\prime}=\frac{\partial \delta}{\partial y_{1}}$

where $k_{4}():.=\frac{k_{k}}{1+\left[s_{1} c_{c}\left(1-s_{1} \delta^{\prime}\right)\right]^{2}}, k_{3}$ and $k_{k}$ are chosen positive, respecting the following conditions:

$k_{3}+\frac{k_{k}}{2}<\frac{R}{L} w_{\max }$,

$u_{\max }:=\frac{R w_{\max }-L / 4}{1+L / 4}$,

$w_{\max }>L / 4 R$,

$u_{d} \leq u_{\max }$

Finally, the wheel velocity control is obtained with:

$w_{\text {left }}=\left(u_{P F}-L r_{P F}\right) / R$,

$w_{\text {right }}=\left(u_{P F}+L r_{P F}\right) / R$,

$\dot{s}=u_{P F} \cos \theta+k_{s} s_{1}$.

Note that $u_{\overline{P F}}=u_{d}$ is achieved when the yaw control $r_{P F}=0$. Moreover, since the surge control $u_{P F}$ can be reduced to zero in case of a strong yaw control $\left(r_{P F}=\right.$ $\left.\frac{R}{L} w_{\max }\right)$, any continuous path is followable, for any value 
of the curvature $c$. This is of course a consequence of the unicycle-type system kinematics. A car-like vehicle does not share this property. This solution is GUAC and respects the constraint (7). The interested reader should refer to Lapierre and Indiverri (2007) for complete proof of convergence.

Proof The proof is organized in two parts. The first one shows that controller (8), where the control gains are chosen according to (10) respects the saturation constraint (7). The second part is showing that this controller also yields GUAC convergence of the robot to the path.

Consider the saturation constraint as expressed in (7). Injecting the control expression (8) in the actuation model (2) provides new expressions for the saturation constraint: $R^{2} w_{\text {max }}^{2}-\left(-L f_{r}+u_{P F}\left(1-L g_{r}\right)\right)^{2} \geq 0$ and $R^{2} w_{\text {max }}^{2}-$ $\left(L f_{r}+u_{P F}\left(1+L g_{r}\right)\right)^{2} \geq 0$. Let's assume that $u_{P F} \geq 0$, then both previous conditions are met if $R^{2} w_{\text {max }}^{2}-\left(L\left|f_{r}\right|+\right.$ $\left.u_{P F}\left(1+L\left|g_{r}\right|\right)\right)^{2} \geq 0$. The analysis of the previous second order inequality easily shows that it is respected if $\left|f_{r}\right|<\frac{R}{L} w_{\max }$. On the other hand, since the expression of $f_{r}$ in (8), one can easily state that $\left|f_{r}\right|<k_{3}+\frac{k_{k}}{2}$. It is then straightforward to see that the respect of the conditions expressed in (10) guarantees that $\left|f_{r}\right|<\frac{R}{L} w_{\max }$, and, as a consequence, the saturation constraints in (7) are respected also.

The second part of the proof shows that the control choice in (8) provides GUAC convergence of the vehicle to the path. Straightforward computation states that the control (8) can be equivalently rewritten as: $r_{\overline{P F}}=\dot{\delta}-k_{3} \tanh (\theta-\delta)+$ $c_{c} \dot{s}$. Considering the Lyapunov candidate $V=\frac{1}{2}(\theta-\delta)^{2}$, in conjunction with the previous expression of the control yields: $\dot{V}=-k_{3} \tanh (\theta-\delta)(\theta-\delta) \leq 0$. Since $V$ is positive definite and $\dot{V}$ is strictly negative if $\theta \neq \delta$, we conclude that $V$ is bounded and has a finite limit as $t$ tends to $\infty$. Moreover, simple derivation shows that $\ddot{V}$ is bounded, that induces that $\dot{V}$ is uniformly continuous. Hence, the necessary requirements for an application of the Barbalat's lemma are met. Then we conclude that the system trajectories converge uniformly to a positive invariant set $\Omega:=\left\{\left(s_{1}, y_{1}, \theta\right)\right.$ : $\theta=\delta\}$. At this point, and because the system is autonomous, we restrict the domain of study to the set $\Omega$. Consider now the Lyapunov candidate $V_{1}=\left.\frac{1}{2}\left(s_{1}^{2}+y_{1}^{2}\right)\right|_{\Omega}$. Clearly the choice for $\dot{s}$ in (11) implies that $\dot{V}_{1}=y_{1} u_{P F} \sin \delta-$ $k_{4}(.) s_{1}^{2} \leq 0$, since the definition of $\delta$. Simple derivation shows that the uniform continuity is related to some trivial conditions, discussed in Lapierre and Indiverri (2007). Finally, the same argument than previously used is invoked to conclude that $\Omega_{1}:=\left(s_{1}, y_{1}, \theta\right): s_{1}=y_{1}=0$ is an invariant set of $\Omega$, and moreover, since the definition of $\delta, \Omega_{1}$ is reduced to the origin. At last, the LaSalle's invariance principle is used in order to show the GUAC convergence of the trajectories to $\Omega_{1}$, that is the origin, i.e. $\left(y_{1}, s_{1}, \theta\right)=0^{3}$.
For a lucid exposition of the control design and the proofs, the reader should refer to Lapierre and Indiverri (2007). The advantages of this solution are:

- The surge velocity is naturally reduced as the heading control increases. As we will see in the sequel, this behavior will be very useful in the obstacle avoidance problems.

- Any continuous path is followable.

- It provides a GUAC convergence of the vehicle to the path.

\subsection{Obstacle avoidance: the safe maneuvering zone (SMZ)}

The objective here is to afford the robot with obstacle avoidance capability, while preserving the GUAC property of the path following controller. The idea is to treat the problem as a Guidance functionality allowing for switching from the path-following target $\left(\mathcal{T}_{P F}\right)$ to the obstacle avoidance target $\left(\mathcal{T}_{O A}\right)$, when necessary.

\subsubsection{The safe maneuvering zone: principle}

The closest proximity sensor information is considered, and the absolute position of the impact (intersection between proximity sensor/obstacle) is computed and stored, and denoted $\mathbf{C}_{O A}$. Note that $\mathbf{C}_{O A}$ is updated only if the closest sensor $k$ provides $d_{k}<\left|\overline{\mathbf{P}_{R} \mathbf{C}_{O A}}\right|$. The SMZ is then defined as $\mathcal{S}_{O A}\left(r_{\mathcal{S}}, \mathbf{C}_{O A}\right)$, where $r_{\mathcal{S}}$ is, for the moment, the arbitrary chosen SMZ's radius.

We introduce a boolean variable $\sigma_{O A}$ according to:

$\sigma_{O A}:=1 \quad$ if $\left[\left(\left|\overline{\mathbf{P}_{R} \mathbf{C}_{O A}}\right|<2 r_{\mathcal{S}}\right) \&\left(\psi_{P F} \in \mathcal{S}_{O A}\right)\right]$, $\sigma_{O A}:=0$ otherwise.

The condition $\left(\psi_{P F} \in \mathcal{S}_{O A}\right)$ means that the ray starting from the robot with absolute direction $\psi_{P F}$ crosses the circle $\mathcal{S}_{O A}$. The first condition in (12), expresses the fact that the closest impact $\mathbf{C}_{O A}$ is inside the SMZ circle. The second condition $\left(\psi_{P F} \in \mathcal{S}_{O A}\right)$ denotes the situation where the global path following heading reference is crossing the SMZ, i.e. the path following controller drives the system within the SMZ. When both conditions are met, obstacle avoidance is necessary, $\sigma_{O A}=1$, and the current reference switches from $\mathcal{T}_{P F}$ to $\mathcal{T}_{O A}$. On the other hand, the system is allowed to disengaged from obstacle avoidance strategy when at least one of the condition is not met.

We also introduce another boolean variable $\Sigma_{O A}$ that records the 'side' (portboard/starboard) where the first impact occurred. This 'side' will be kept until the necessity for obstacle avoidance vanishes (i.e. $\sigma_{O A}=0$ ). Consider that the first closest impact has been given by the sensor $k$, then

$$
\begin{array}{ll}
\Sigma_{O A}:=1 & \text { if }\left[\left(\alpha_{k} \in[0, \pi]\right) \&\left(\sigma_{O A}=1\right)\right], \\
\Sigma_{O A}:=2 & \text { if }\left[\left(\alpha_{k} \in[\pi, 2 \pi]\right) \&\left(\sigma_{O A}=1\right)\right], \\
\Sigma_{O A}:=0 & \text { otherwise. }
\end{array}
$$


Then, if $\sigma_{O A}=1, \mathcal{T}_{O A}$ exists, and $\psi_{O A}$ is chosen as one of the two directions of the tangent of $\mathcal{S}_{O A}$ on $\mathcal{T}_{O A}$. The direction of $\overrightarrow{\mathbf{P}_{R} \mathbf{C}_{O A}}$ is computed as $\psi_{\overline{R C}}:=\operatorname{atan} 2\left(\left(y_{O A}-y\right)\right.$, $\left.\left(x_{O A}-x\right)\right)$, where atan2() is the four quadrants arctangent computation function. The $\psi_{O A}$ direction is chosen according to

$$
\begin{array}{ll}
\psi_{O A}:=\psi_{\overline{R C}_{O A}}+\pi / 2, & \text { if }\left[\Sigma_{O A}=1\right], \\
\psi_{O A}:=\psi_{\overline{R C}_{O A}}-\pi / 2, & \text { if }\left[\Sigma_{O A}=2\right] .
\end{array}
$$

This particular choice induces an interesting behavior. When the first detection is made on portboard (obstacle on the left side, $\Sigma_{O A}=1$ ), the choice $\psi_{O A}=\psi_{\overline{R C}_{O A}}+\pi / 2$ the system is contouring the obstacle by the right, and keep this strategy as fas as $\sigma_{O A}=1$. Similar strategy occurs when the initial detection is made on starboard. This is allowing for avoiding the known trap situation, called corner situation in Lapierre et al. (2007), where obstacles are present on both sides. This computation of $\psi_{O A}$ induces a U-turn in such a situation. This implies that the ability of the vehicles to enter and maneuver into a narrow corridor, for example, depends on the size of the SMZ, i.e. $r_{\mathcal{S}}$.

Moreover, an unbounded definition of the angles involved in the computation of the second condition of (12), i.e. $\left\{\psi, \psi_{P F}, \psi_{O A}\right\} \in[-\infty,+\infty]$, induces another interesting behavior. Since the vehicles are 'keeping the contact' with obstacles on the same side (portboard/starboard), they naturally find an issue from the entrance to the exit of a $2 \mathrm{D}$ maze, if the solution exists and, consecutively, contour the obstacles to converge again to the path, when $\sigma_{O A}=0$. As a consequence a solution exists if there is a continuous region, free of obstacle, with a minimum width of $2 r_{\mathcal{S}}$.

Finally, the control is achieved in considering $\mathcal{T}_{O A}$ and $1 / r_{\mathcal{S}}$ instead of $\mathcal{T}_{P F}$ and $c$, within the control expression (11).

Now an interesting question arises: what performances are expected from this obstacle avoidance strategy? In the following, we propose a method to chose the size of the $\mathrm{SMZ}, r_{s}$, such that the induced maneuver is guaranteed to be performed within a global amplitude less than $r_{s}$.

\subsubsection{Choice of $r_{s}$}

The aim of the following propositions is to find a method to choose the control gains and $r_{s}$, in order to guarantee that the avoidance is safely performed. This is achieved using a set of conditions, exposed in the following statements. The objective is to find a feasible solution. Optimization comes after.

Some preliminary remarks have to be exposed. Once the obstacle avoidance strategy is engaged, the robot converges to $\mathcal{T}_{O A}$, which lives on the SMZ boundary, i.e. a circle of radius $r_{s}$. By construction $\mathbf{C}_{O A}, \mathbf{P}_{R}$ and $\mathcal{T}_{O A}$ are aligned. We conclude that $s_{1}=0$ and $y_{1}=\left\|\mathbf{C}_{O A} \mathbf{P}_{R}\right\|$. Let denote the initial instant when the obstacle avoidance is engaged as $t_{O A}^{0}$, then $y_{1}\left(t_{O A}^{0}\right)=0$, and let $\tilde{\theta}^{0}=\theta\left(t_{O A}^{0}\right)-\delta\left(t_{O A}^{0}\right)$ denotes the initial angle of incidence of the robot with respect to the SMZ tangent.

Proposition 2 The controller exposed in (8) induces trajectories that remain within the maneuvers induced by the unsaturated version of the path following controller (5). That $i s$, given a system situation $\left(y_{1}, s_{1}, \theta\right)$, the resulting saturated control computation (8) induces a trajectory with a higher curvature than with (5), i.e.:

$\left|\frac{r_{\overline{P F}}}{u_{\overline{P F}}}\right|>\left|\frac{r_{P F}}{u_{d}}\right|, \quad \forall t>t_{O A}^{0}$.

In other words, the use of (8) does not drive the system closer to the obstacle, than using (5). The rationale of this argument is to exploit the GEC property of (5), which induces maneuvers that bound the trajectories induced with (8). Hence, proving that $y_{1}(t)<r_{s}-B_{S}, \forall t>t_{O A}^{0}$, where $B_{S}$ plays the role of a security margin, with (5) is enough to prove that the system remains at a minimum distance of $B_{S}$ from obstacle, using (8).

Proof The idea is to find the condition on the choice of the control gains, $K_{2}$ and $k_{3}$, such that the curvature of the trajectories induced by (5) is always smaller than the one obtained with (8). This is expressed by condition (15).

Using the expression of (5) and (8), and their respective developments, we can equivalently rewrite Condition (15) as:

$a . b . k_{3} \tanh (|\theta-\delta|)>K_{2}(|\theta-\delta|)$

where $a=\frac{1+\frac{1}{4}+L f_{r}^{2}}{1+\frac{1}{4}}$ and $b=\frac{R w_{\max }-\frac{L}{4}}{R w_{\max }-\frac{L}{4}-L g_{r}^{2}}$, which under the conditions (10) are both bigger than 1 . When obstacle avoidance is engaged, the maximum value achieved by $\theta-\delta$ is $\pi / 2$, hence (16) is true if:

$k_{3}>K_{2}\left(1+\frac{\pi^{2}}{4}\right)$.

Conditions (10) have also to be considered, which with obstacle avoidance particularity $\left(s_{1}=0\right)$ can be expressed as $k_{3}<\frac{R}{L} w_{\max }$. Then global condition on the gain $k_{3}$, expressed as:

$\frac{R}{L} w_{\max }>k_{3}>K_{2}\left(1+\frac{\pi^{2}}{4}\right)$,

restricts the range of admissible values for $k_{3}$.

Since the previous argument, we can now use the exponential convergence property of the guidance error $(\theta-\delta)$, induced by the control (5),

$$
\begin{aligned}
& (\dot{\theta}-\dot{\delta})=-K_{2}(\theta-\delta) \quad \Rightarrow \\
& \theta(t)=\tilde{\theta}_{0} e^{-K_{2} t}+\delta(t), \quad \forall t>t_{O A}^{0} .
\end{aligned}
$$


Proposition 3 The control (5) induces a bounded behavior of $y_{1}$, for which an upper bound $Y^{\max }>\left|y_{1}\right|$ is computable.

Proof Combining (3) and (19) with the following particular definition for the approach angle:

$\delta=-\arctan \left(k_{\delta} y_{1}\right)$

yields to the differential relation:

$\dot{y}_{1}=\frac{u_{d}}{\sqrt{1+\left(k_{\delta} y_{1}\right)^{2}}}\left(\sin \left(\tilde{\theta}_{0} e^{-K_{2} t}\right)-\cos \left(\tilde{\theta}_{0} e^{-K_{2} t}\right) k_{\delta} y_{1}\right)$.

Since all the multiplicative terms are bounded, it is clear that $y_{1}$ is bounded, as expected. In the absence of other criterion to consider (e.g. energy consumption) the highest rate of convergence is obtained with $k_{\delta}$ big, and $K_{2}$ maximum, i.e. $K_{2}=\frac{R w_{\max }}{L}$. Recall that $k_{\delta}$ defines the slope of the system approach to the path. Hence, a high value for $k_{\delta}$ induces a perpendicular approach to the path for small values of $y_{1}$. In the discretized environment of the processor onto which the algorithm is implemented, this choice may induce an undesired chattering behavior. This point has to be deeper investigated. The computation of the upper bound $y_{1}^{\max }$ is performed using a numerical resolution of $\frac{\dot{y}_{1}}{u_{d}}$, for different values of $k_{\delta} \in[0.1,10]$ and $K_{2}=\frac{R w_{\max }}{L}$. Given system parameters, e.g. $R=0.1 \mathrm{~m}, L=0.25 \mathrm{~m}$ and $w_{\max }=10 \pi \mathrm{rad} / \mathrm{s},(21)$ is solved, and the maximum value of $y_{1}^{\max }\left(k_{\delta}\right) / u_{d}$ is computed and stored. Figure 4 shows the expected maximum penetration of the system within the SMZ, i.e. $y_{1}^{\max } / u_{d}$, versus the guidance's gain of the desired approach, i.e. $k_{\delta}$. Clearly the best behavior is achieved for big $k_{\delta}$, and admits a lower bound. This lower bound corresponds to the limit situation for $k_{\delta} \rightarrow \infty$, where the dynamics of the intrusion responds to $\left.\dot{y}_{1}\right|_{k_{\delta} \rightarrow \infty}=-\cos \left(\tilde{\theta}_{0} e^{-K_{2} t}\right)$. Hence, given system parameters $R, L$ and $w_{\max }$, the slope of the desired approach $k_{\delta}$ and the desired forward velocity $u_{d}$, an upper bound to the maximum intrusion is computable, referring to Fig. 4, and $Y^{\max }=\left[\frac{y_{1}^{\max }}{u_{d}}\right]\left(k_{\delta}\right) u_{d}$.

Hence, given a safety margin $B_{S}$, the radius of the SMZ is chosen as:

$r_{s}=Y^{\max }+B_{S}$.

\subsubsection{Some illustrative simulations}

We illustrate the performances of the solution using the path depicted at Fig. 5. The considered system and control parameters are given at Table 1 considered, where $k_{3}<$ $\frac{R w_{\max }}{L} \frac{1}{1+\frac{\pi^{2}}{4}}$, according to the previous argument.

The evolution of the system velocities are drawn at Fig. 6 and indicates the respect of the saturation constraint (7).

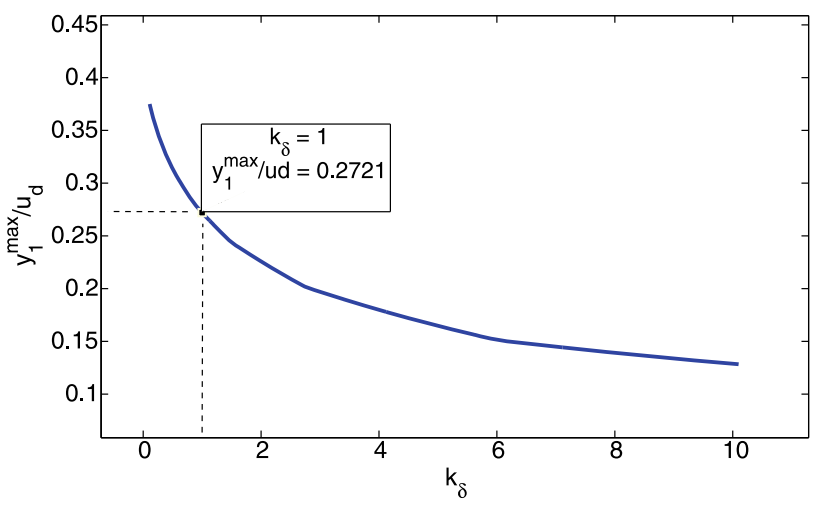

Fig. 4 Maximum penetration within the SMZ, versus the approach parameter $k_{\delta}$

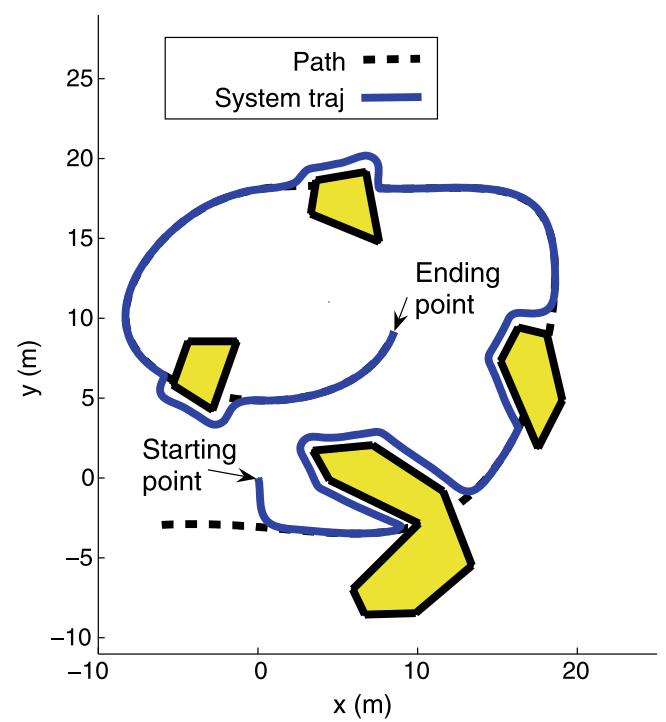

Fig. 5 System trajectory

Table 1 System and control parameters

\begin{tabular}{lll}
\hline$R=0.1 \mathrm{~m}$ & $L=0.25 \mathrm{~m}$ & $w_{\max }=10 \cdot \pi$ \\
\hline$k_{3}=3.5$ & $k_{\delta}=1$ & $k_{1}=1$ \\
\hline
\end{tabular}

Finally, Fig. 7 shows the evolution of the variables $y_{1}$ and the minimum distance to the obstacles, when obstacle avoidance strategy is engaged. Clearly the system does not get closer to the obstacle than $B_{S}=0.5 \mathrm{~m}$, and the maximum penetration within the DVZ does not exceed the expected bound, i.e. $Y_{\max }=0.2721 \cdot u_{d}=0.5442 \mathrm{~m}$.

Figures exposed in Fig. 8 decompose the system trajectory with respect to some particular instants. Figure 8(a) illustrates the system behavior, approaching the desired path and tracking the path following virtual target $\mathcal{T}_{P F}$, far from any obstacle. Figure $8(\mathrm{~b})$ shows the first instant when the proximeters detect an obstacle, at point denoted $C_{O A}$. The $\operatorname{SMZ}\left(\mathcal{S}_{O A}\right)$, centered in $C_{O A}$ with radius $r_{\mathcal{S}}$, is built. Next 



Fig. 6 System velocities
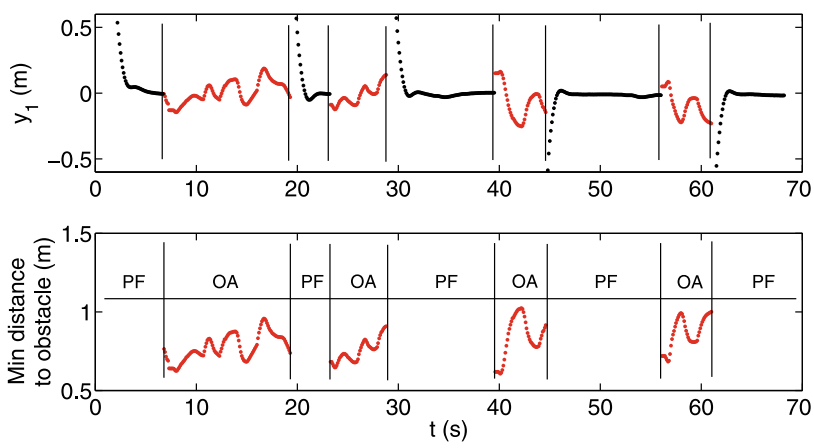

Fig. 7 Evolution of $y_{1}$, and minimum distance to obstacle

Fig. 8(c) shows the evolution of the new virtual target $\mathcal{T}_{O A}$, living on $\mathcal{S}_{O A}$ and positioned on the intersection of $\mathcal{S}_{O A}$ and $\overline{\mathbf{P}_{R} \mathbf{C}_{O A}}$. Note that the path following virtual target $\mathcal{T}_{P F}$ continues its progression on the path, converging to the closest point between the robot and the path, as expected with the path following virtual target controller (11). Figure 8(d) shows a change in $C_{O A}$, while another part of the obstacle is getting closer. Note the orientation of the absolute path following heading $\psi_{P F}$ that still crosses the $\mathcal{S}_{O A}$, that keeps the obstacle avoidance behavior engaged. The vehicle is then following the obstacle, as shown at Fig. 8(e). In fact, in this situation, the system is memorizing the actual $C_{O A}$, which is updated only if a closer detection occurs. If for any reason, the obstacle vanishes (e.g. dynamic obstacle) the system will keep considering the actual $C_{O A}$ and turn around it until the condition of disengagement form Obstacle Avoidance to Path Following is filled. This behavior is illustrated Fig. 8(f), where the robot is contouring a salient angle of the obstacle. In this situation, the desired system trajectory is a portion of the circle $\mathcal{S}_{O A}$. Figure $8(\mathrm{~g})$ shows the instant when the orientation of the path following desired heading $\psi_{P F}$ leaves the circle $\mathcal{S}_{O A}$, consequently disengaging the Obstacle Avoidance behavior, and tracking now the path following virtual

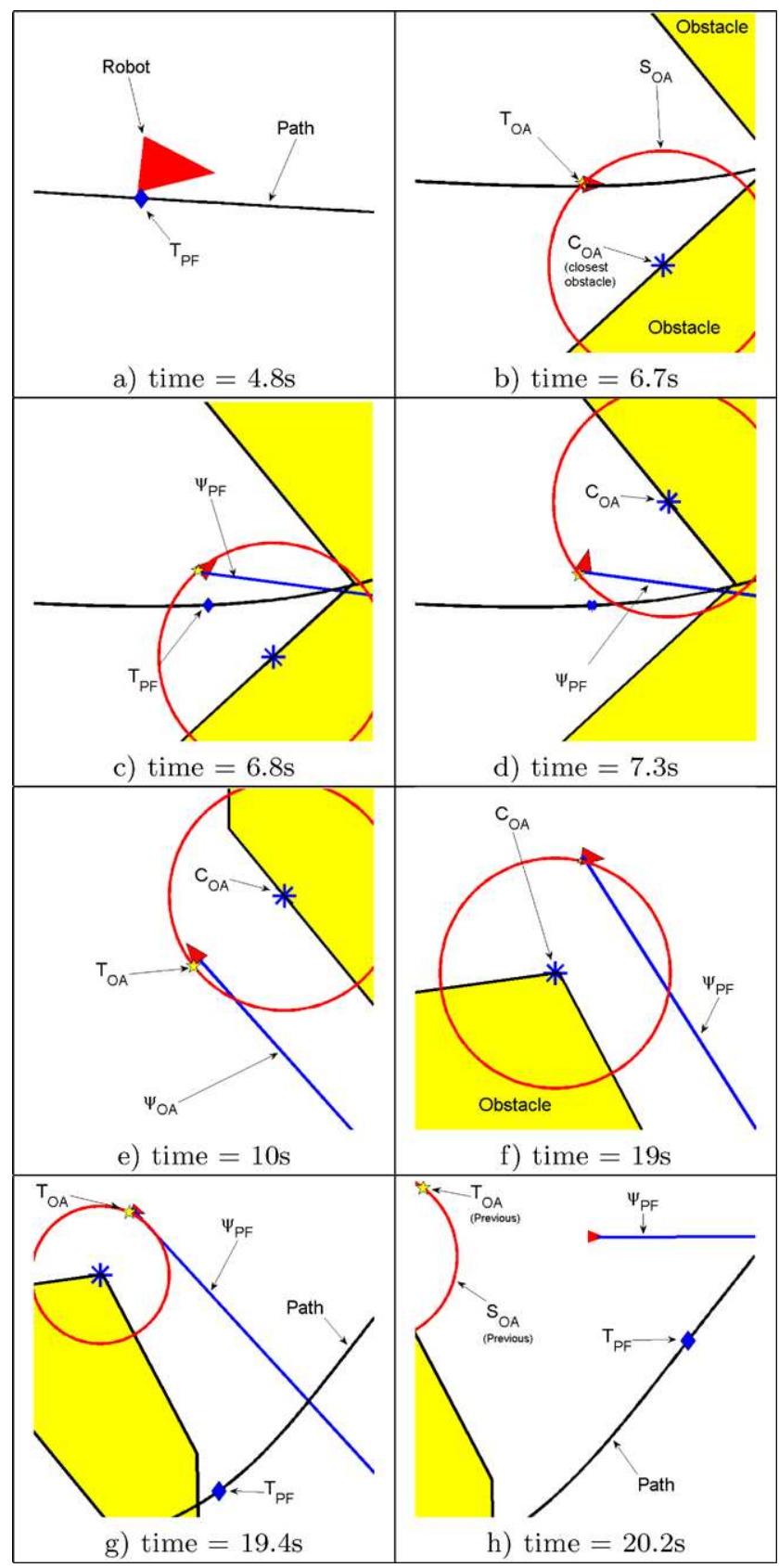

Fig. 8 Snapshots of the simulation

target $\mathcal{T}_{P F}$. Note that during the Obstacle Avoidance behavior, the path following virtual target was still continuing its progression on the path. Then, as described at Fig. 8(h) the robot meets the path following virtual target $\mathcal{T}_{P F}$, after having contoured the obstacle.

\section{Conclusion}

This paper proposes a solution to the control of movement of a unicycle-type robot, including path-following and obstacle avoidance, while respecting the actuation saturation 
constraint. The goal is to provide a solution with guaranteed performances. That is, in our context, to drive the system to globally, uniformly and asymptotically converge to the path, when possible, and contour the unpredicted obstacles, respecting a minimum distance between the robot and the obstacle. This guaranteed minimum distance is a function of the system capability, in terms of actuation saturation constraint. Illustrative simulated examples are given.

An extension of this work will use the virtual target principle to generalize the method to multiple-vehicles system, including collision avoidance capability, in the presence of communication delay in the necessary information to exchange within the formation.

\section{References}

Aguiar, A. P., Atassi, A., \& Pascoal, A. M. (2000). Regulation of a nonholonomic dynamic wheeled mobile robot with parametric modeling uncertainty using Lyapunov function. In The Proceeding of CDC 2000, 39th IEEE conference on decision and control, Sydney, Australia.

Arrichiello, F., Chiaverini, S., Pedone, P., Zizzari, A., \& Indiveri, G. (2009). The null-space based behavioral control for nonholonomic mobile robots with actuators velocity saturation. In The proc. of the IEEE international conference on robotics and automation (pp. 4019-4024), Kobe, Japan.

Astolfi, A. (1999). Exponential stabilization of a wheeled mobile robot via discontinuous control. Journal of Dynamics, Systems, Measurement and Control, 121, 121-126.

Brockett, R. W. (1983). Asymptotic stability and feedback stabilization. In R. W. Brockett, R. S. Millman, H. J. Sussman (Eds.), Differential geometric control theory (pp. 181-191). Boston: Birkhäuser.

Canudas de Wit, C., \& Sordalen, O. (1992). Exponential stabilization of mobile robots with nonholonomic constraints. IEEE Transactions on Automatic Control, 37, 1791-1797.

Canudas de Wit, C., Khennouf, H., Samson, C., \& Sordalen, O. (1993). Nonlinear control design for mobile robots. In Y. F. Zheng (Ed.), World scientific series in robotics and automated systems: Vol. 11. Recent trend in mobile robots.

Elnagar, A., \& Hussein, A. (2002). Motion planning using Maxwell's equations. In The proc. of the IEEE/RSJ international conference on intelligent robots and systems, Lausanne, Switzerland.

Encarnacao, P., Pascoal, A., \& Arcak, M. (1992). Path following for autonomous marine craft. In Proceedings of the 5th IFAC conference on marine craft maneuvering and control, MCMC'00 (pp. $117-$ 122), Aarlborg, Denmark.

Fierro, R., \& Lewis, F. (1994). Control of a nonholonomic mobile robot: backstepping kinematics into dynamics. In Proceedings of the 33rd conference on decision and control, Florida, USA.

Freund, E., \& Mayr, R. (1997). Nonlinear path control in automated vehicle guidance. IEEE Transactions on Robotics and Automation, 13(1), 49-60.

Ge, S., \& Cui, Y. (2000). Path planning for mobile robots using new potential functions. In Proc. of the 3rd Asian control conference, July 4-7, Shanghai, China.

Godhavn, J. M., \& Egeland, O. (1997). A Lyapunov approach to exponential stabilization of nonholonomic systems in power form. IEEE Transactions on Automatic Control, 42(7), 1028-1032.

Hespanha, J. P. (1996). Stabilization of nonholonomic integrators via logic based switching. In The proceedings of the 13th world congress of IFAC (Vol. E, pp. 467-472), San Francisco, CA, USA.
Indiveri, G., Paulus, J., \& Ploger, P. G. (2009). Motion control of Swedish wheeled mobile robots in the presence of actuator saturation. In Lecture notes in computer science (Vol. 4434, pp. 35-46). doi:10.1007/978-3-540-74024-74.

Iniguez, P., \& Rossel, J. (2002). A hierarchical and dynamic method to compute harmonic functions for constrained motion planning. In The proc. of the IEEE/RSJ international conference on intelligent robots and systems, Lausanne, Switzerland.

Jiang, Z., \& Nijmeijer, H. (1999). A recursive technique for tracking control of nonholonomic systems in the chained form. IEEE Transactions on Automatic Control, 44(2), 265-279.

Jiang, Z. P., Lefeber, E., \& Nijmeier, H. (1998). Stabilization and tracking of a nonholonomic mobile robot with saturating actuators. In The proc. the third Portuguese conference on automatic control (CONTROLO'98), Coimbra, Portugal.

Krstić M., Kanellakopoulos I., \& Kokotovic P. (1995). Nonlinear and adaptive control design. New York: Wiley.

Lapierre, L., \& Indiverri, G. (2007). Path-following control of a wheeled robot under actuation saturation constraints. In Proceedings of the IAV'07 conference, Toulouse, France.

Lapierre, L., Soetanto, D., \& Pascoal, A. (2006). Nonsingular path following control of a unicycle in the presence of parametric modeling uncertainties. International Journal of Robust and Nonlinear Control, 16, 485-503. doi:10.1002/rnc.1075.

Lapierre, L., Zapata, R., \& Lepinay, P. (2007). Combined path following and obstacle avoidance control of a wheeled robot. The International Journal of Robotics Research, 26(4), 361-375. doi:10.1177/0278364907076790.

Lapierre, L., Zapata, R., \& Bibuli, M. (2010). Guidance of a flotilla of wheeled robots: a practical solution. In Proceedings of the IAV' 10 conference, Lecce, Italy.

Louste, C. (1999). Conception d'une methode de planification pour robot mobile selon la methode des milieux continus appliquee aux fluides visqueux. Ph.D. Thesis nb 6701, LIRMM, Montpellier (1999) (in French).

Micaelli, A., \& Samson, C. (1992). Path following and time-varying feedback stabilization of a wheeled robot. In The Proceedings of the international conference ICARCV'92, Singapore.

Micaelli, A., \& Samson, C. (1993). Trajectory tracking for unicycle type and two steering wheels mobile robots (Technical Report No. 2097). Sophia-Antipolis: INRIA.

Samson, C., \& Ait-Abderrahim, K. (1991). Mobile robot control. Part 1: Feedback control of a non-holonomic mobile robots (Technical Report No. 1281). Sophia-Antipolis, France: INRIA.

Sgorbissa, A., Capezio, F., Zacaria, R., Rebora, A., \& Campani, M. (2010). A minimalist approach to path-following among unknown obstacles. In The proc. of the IEEE international conference on intelligent robots and systems, Taipei, Taiwan.

Walsh, G., Tilbury, D., Sastry, S., \& Laumond, J. P. (1994). Stabilization of trajectories for systems with nonholonomic constraints. IEEE Transactions on Automatic Control, 39(1), 216-222.

Zapata, R., Cacitti, A., \& Lepinay, P. (2004). DVZ-based collision avoidance control of non-holonomic mobile manipulators. JESA, European Journal of Automated Systems, 38(5), 559-588. 


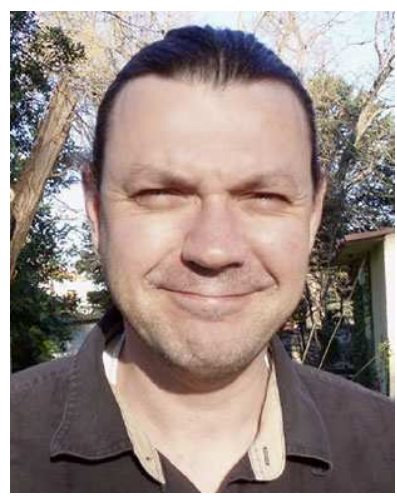

Lionel Lapierre received his Ph.D. degree in Robotics, from the University of Montpellier 2, Montpellier, France, in 1999. Then, he joined the team of Professor A. Pascoal within the European project FreeSub for three years. Since 2003, he has been with the Underwater Robotics Division, Laboratoire d'Informatique, de Robotique et de Microélectronique de Montpellier (LIRMM), Montpellier, France.

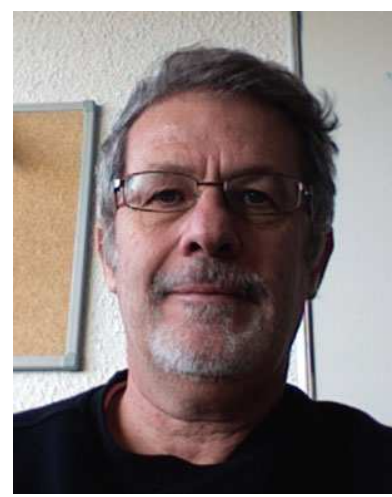

Rene Zapata is professor at the University Montpellier II (FRANCE) in the Laboratoire d'Informatique, de Robotique et de Microélectronique de Montpellier (LIRMM UMR 5506) since 1983. He works in the Robotics Department of LIRMM in the fields of Robot Control, Motion Planning and Path Planning, Robot Cooperation and Robot Localization applied to mobile robots (ground robots, flying robots and humanoids). $\mathrm{He}$ is interested in the non-linear control of mobiles robots and more especially in the design of algorithms for controlling the reactive behaviors of multi-robots moving in unknown and dynamic environments. $\mathrm{He}$ teaches Theory of control, mathematics and robotics at Master level at the university Montpellier II. 